\title{
The Management of Children with Gastroesophageal Reflux and Respiratory Symptoms
}

\author{
Usha Krishnan ${ }^{1}$, Shaun Rama ${ }^{2}$, Isabella Messina ${ }^{3}$ and Emily Horsley ${ }^{4}$ \\ ${ }^{1,3,4}$ Department of Paediatric Gastroenterology, Sydney \\ ${ }^{1,2,3,4}$ School of Women's and Children's Health, University of New South Wales, Sydney, Australia
}

Correspondence should be addressed to: Usha Krishnan; usha.krishnan@sesiahs.health.nsw.gov.au

Received date: 8 July 2014; Accepted date: 4 September 201; Published date: 27 April 2015

Academic Editor: Yván Vandenplas

Copyright (C) 2015. Usha Krishnan, Shaun Rama, Isabella Messina and Emily Horsley. Distributed under Creative Commons CC-BY 4.0

\begin{abstract}
Research Motivation: Gastroesophageal reflux disease (GERD) is a well-recognised cause of respiratory symptoms in children. Confirming a diagnosis of reflux aspiration remains difficult, due to limitations in currently available investigations. The presence of pepsin in respiratory secretions has been documented in literature as a marker of reflux aspiration; however the correlation of pepsin assay results with clinical outcomes has not previously been reported. Aim: This study investigates the way in which results of pepsin assays influence the management of children with suspected reflux aspiration, as well as clinician attitudes towards pepsin assay. Methodology: Retrospective chart review of 318 patient records from January 2005 - December 2009, at Sydney Children's Hospital. Pepsin assay results were compared with results from other standard investigations for GERD. Findings: Children with GERD and respiratory symptoms were 1.8 times as likely to be positive for pepsin compared to children without respiratory symptoms (OR 1.8, 95\% C.I. $=1.09-2.96$ ). A significantly greater quantity of pepsin was detected in tracheal aspirates of children with GERD and respiratory symptoms compared to those without (median 43, 0-996FU, $\mathrm{p}=0.002$ ). In children with GERD and respiratory symptoms, pepsin assay, unlike standard GERD investigations was the only tool found to have statistical significance as a marker of possible reflux aspiration. Clinicians found the pepsin assay to be useful in the management of children with suspected reflux aspiration. Implications: The findings of this study show that the pepsin assay helps in the assessment of children with GERD and respiratory symptoms in who reflux aspiration are suspected.
\end{abstract}

Keywords: pepsin; reflux; aspiration; GERD

Cite this Article as: Usha Krishnan, Shaun Rama, Isabella Messina and Emily Horsley (2015)," The Management of Children with Gastroesophageal Reflux and Respiratory Symptoms", International Journal of Gastroenterology Research and Practice, Vol. 2015 (2015), Article ID 247821, 


\section{Introduction}

Gastroesophageal reflux disease (GERD) is a well-recognised cause of respiratory symptoms in children and is known to exacerbate pre-existing respiratory disease $^{1 .}$ The respiratory system is affected in a range of ways including bronchoconstriction, pneumonia, asthma, otolaryngotic infections, apnoea, chronic nasal congestion and chronic cough ${ }^{2} .60$ $70 \%$ of children have experienced at least one episode of prolonged esophageal exposure to refluxate by the age of 4 months, which can lead to complications such as chronic respiratory disease ${ }^{3}$. The detection of pepsin in respiratory and otolaryngologic secretions has been reported in the literature as accurate markers for reflux aspiration as they are not normally found in the respiratory tract and their presence indicate the aspiration of gastric contents ${ }^{4}, 5$. The author ${ }^{3}$ was one of the first to report that the use of pepsin assay in conjunction with standard investigations helps to more accurately diagnose reflux aspiration, in comparison to the use of standard investigations for GERD alone. In this study of children with reflux symptoms and respiratory disease, the authors found $84 \%$ of patients with respiratory disease and reflux symptom were pepsin positive. In contrast, $87 \%$ of children with respiratory disease but no reflux symptoms were pepsin negative. These data suggest that reliance on symptoms for the determination of reflux related respiratory disease, the use of $\mathrm{pH}$ monitoring to detect acid reflux alone or the use of gastroscopy to detect oesophagitis alone are imperfect for the establishment of causality between GERD and respiratory symptoms. Since then, several papers have commented on the role of pepsin as a marker of reflux aspiration including the recent joint ESPGHAN and NASPGHAN guidelines on the management of GERD in children ${ }^{20}, 21,22,23,24,25,26$. However the correlation of pepsin assay with clinical management and outcomes has not previously been formally explored. This study investigates the way in which pepsin assays influence patient management and clinician attitudes towards pepsin assay within paediatric medicine.

\section{Methods}

\section{Study Design}

Three hundred and eighteen patient charts from 2007 - 2009 were reviewed by the Sydney Children's Hospital in a retrospective chart review. All patients had symptoms suggestive of gastro esophageal reflux and some of these patients also had respiratory symptoms. Patients included had either laryngobronchoscopy (LB) or gastroscopy. Patients underwent tracheal aspirate pepsin assays whilst endotracheally intubated for these investigations.

\section{Investigation Methods}

Respiratory secretions were collected during LB or whilst the children were intubated for gastroscopy, after instilling $2 \mathrm{mls}$ of normal saline and suctioning into a sputum trap. The pepsin assay utilised was modified from the Twining 6 assay using fluorescein isothiocyanate casein (FITC). Information was collected about stand tests done to investigate GERD including $\mathrm{pH}$ probes, gastroscopy and barium meal. $\mathrm{pH}$ probe results were interpreted using the Vandenplas \& Sacre-Smith ${ }^{7}$ criteria and categorised as normal or abnormal. Gastroscopy was performed to exclude reflux esophagitis or eosinophilic esophagitis. Biopsies were considered abnormal if the biopsies showed evidence of reflux esophagitis as per the LA classification $^{28}$. If the eosinophil count measured more than 20 in any high powered field, then a diagnosis of Eosinophilic Esophagitis was made ${ }^{29}$. Barium contrast studies were considered abnormal if reflux episodes were noted during the study. This modality is recognised as being neither a sensitive nor a specific marker for reflux disease, however it is widely used in the Australian hospital setting and results were included for completeness. Data were also collected on the medical and surgical treatments received by these patients for treatment of gastroesophageal reflux disease. Patients 
were categorised as having chronic respiratory symptoms if they had persistent cough, asthma, recurrent bronchitis or recurrent pneumonia.

\section{Questionnaire}

Questionnaires were completed by treating clinicians to assess their attitudes towards pepsin assays and the usefulness of pepsin assay results in altering their clinical management. Clinicians responding to the questionnaire included specialists from the fields of pediatric gastroenterology, general pediatrics, intensive care, pediatric respiratory medicine and Ear, Nose and Throat surgery. Details of questionnaires sent to the primary clinician involved in each patient's care are shown below.

Question 1: $\quad$ Did you find the results of the pepsin assay to be helpful?

Question 2: Did the results of the pepsin assay lead to an alteration in the treatment of your patient?

Question 3: Did the results of the assay result in alteration of the anti-reflux medications that the patient was on?

Question 4: Did the results of the assay result in a fundoplication being performed on the patient?

Question 5: Did you obtain any new diagnostic information this assay which was not previously available?

\section{Statistical Analysis}

An Excel database was created to enter the data collected, and the results of standard GERD investigations and treatments were correlated with the results of pepsin assay.

Results were entered into an SPSS statistical software (Version 17, SPSS Inc, Chicago, IL); database and relevant statistics and graphs were calculated using the Chi squared test and Fisher exact test. A significance level of $p<0.05$ was chosen for statistical analysis.

\section{Ethics}

Ethics approval was received from the Research and Ethics Committee of the South Eastern Sydney Area Health Service.

\section{Results}

\section{Pepsin assay}

Patients were between the ages of 4 months and 13 years of age, $60 \%$ of patients were male. The mean age of children in the study was 3 years, 7 months with a median age of 2 years, 5 months as shown in Figure 1.

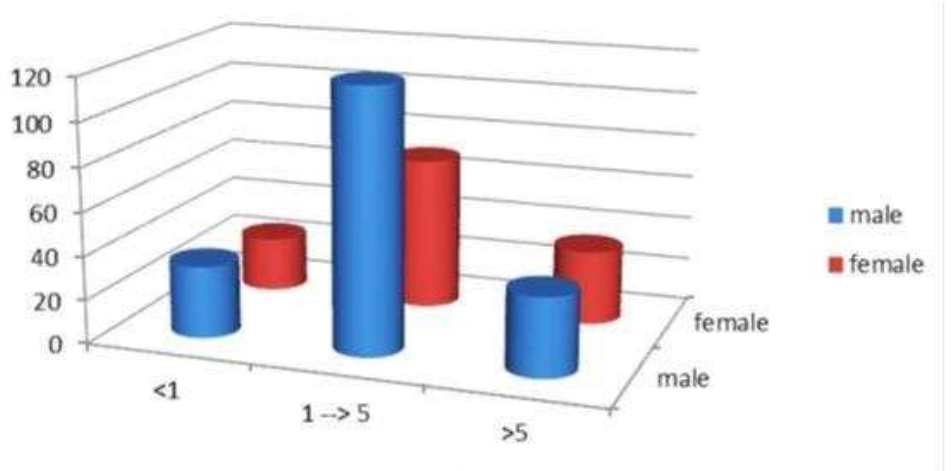

Figure 1: Patient demographics by age and gender

Figure 1: Patient demographics by age and gender 
Of the 318 patients, 111 patients had sampling of their respiratory secretions done during LB and the remaining had a tracheal aspirate collected post intubation for other procedures including gastroscopy in 83 patients. 160 patients (50\%) tested positive for pepsin. Of those who had LB, $76 \%$ were pepsin positive, whilst $36 \%$ were positive for pepsin in those who had gastroscopy. The percentage of pepsin positivity was significantly higher in those undergoing LB compared to those undergoing gastroscopy (O.R 5.4, p $<0.05$, 95\% C.I 3.3 - 9.7).

\section{Standard Investigations}

Twenty four hour pH probe monitoring was performed in 64 patients, gastroscopy in 83_patients and barium studies in 44 patients. Results of these investigations are summarised in Table 1.

Table 1: Results of standard investigations for gastroesophageal reflux disease

\begin{tabular}{|l|l|l|l|}
\hline Investigations & $\begin{array}{l}\text { Number of } \\
\text { patients } \\
\text { undergoing } \\
\text { investigation }\end{array}$ & $\begin{array}{l}\text { Number of } \\
\text { abnormal } \\
\text { results }\end{array}$ & $\begin{array}{l}\text { Percentage of } \\
\text { abnormal } \\
\text { results }\end{array}$ \\
\hline pH Probe & 64 & 11 & $17 \%$ \\
\hline Endoscopy & 83 & 32 & $38 \%$ \\
\hline Barium Meal & 44 & 11 & $25 \%$ \\
\hline
\end{tabular}

In children positive for pepsin in tracheal aspirates, there was no significant difference in the prevalence of positive versus negative results on 'standard'

investigations for GERD as shown in Figure Two.

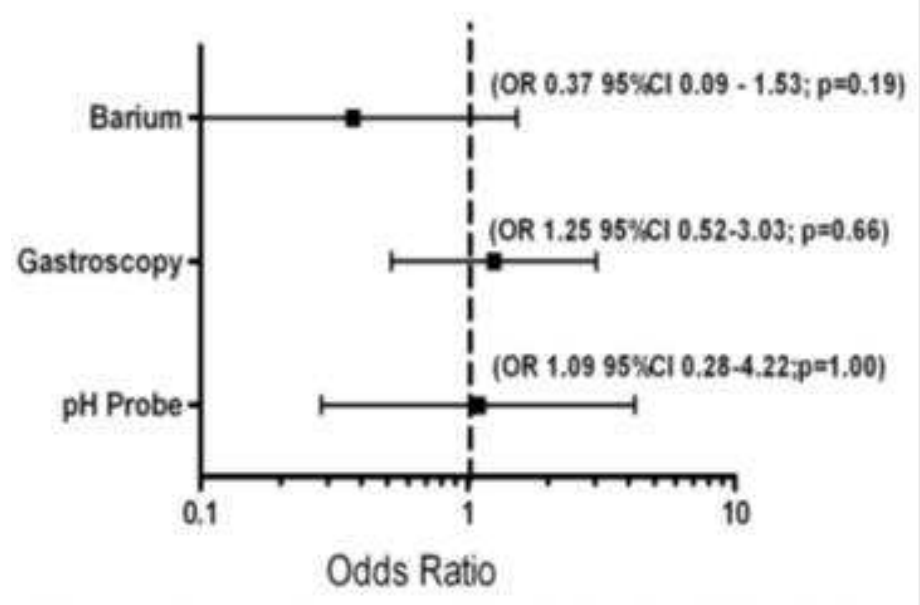

Figure 2: Correlation between positive Pepsin Assay and results of standard investigations for GERD

\section{Figure 2: Correlation between positive pepsin assay and results for standard investigations for GERD}

Information regarding anti reflux therapy was available in 194 of the 318 patients studied. $45(23 \%)$ of these patients were on antireflux medications at the time of respiratory secretion sampling. 206 patients had information collected regarding fundoplication. Eighteen (8.7\%) of these patients had undergone 
fundoplication prior to tracheal aspirate sampling for pepsin assay. These 18 patients were being investigated for recurrence of reflux and respiratory symptoms. $8(44 \%)$ of these 18 patients tested positive on pepsin assay despite previous fundoplication procedure and 5 of these patients had signs of oesophagitis on endoscopy and biopsy. Only 6 of these 8 patients had $\mathrm{pH}$ probe studies which were all normal (Figure three).

\section{Pepsin Results compared with respiratory symptomatology}

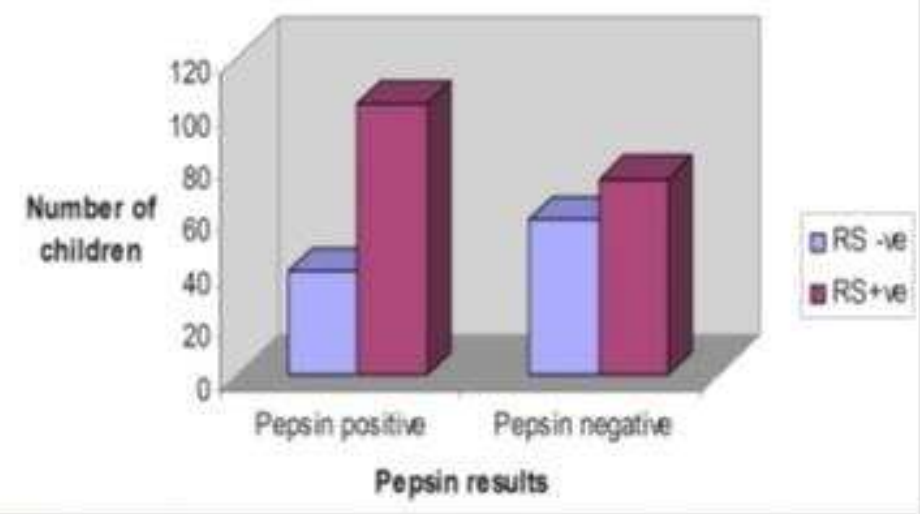

Figure 3: Comparison of Pepsin Assay results with presence of respiratory symptoms

Figure 3: Comparison of Pepsin assay results with presence of respiratory symptoms

\section{Respiratory Symptoms}

Of the 318 patients, $88 \%$ had data collected on the presence of chronic respiratory symptoms. Of these patients, 178(63\%) reported respiratory symptoms occurring within the three month period preceding tracheal aspirate sampling. 58\% of those with positive pepsin assay had concomitant respiratory symptoms, whereas $40 \%$ of those with negative pepsin assay had respiratory symptoms $(\mathrm{p}=0.023)$. Children with respiratory symptoms were 1.79 times more likely to be pepsin positive when compared to children without respiratory symptoms (OR 1.8, 95\% C.I. = $1.09-2.96)$.

When comparing positive pepsin assay results in children with respiratory symptoms in the whole group to the group of children without respiratory symptoms but with reflux symptoms alone, pepsin was significantly more likely to be detected in children with respiratory symptoms (OR 1.79, 95\% CI 1.09-2.96. $\mathrm{p}=0.02$ ) (Figure Four). 


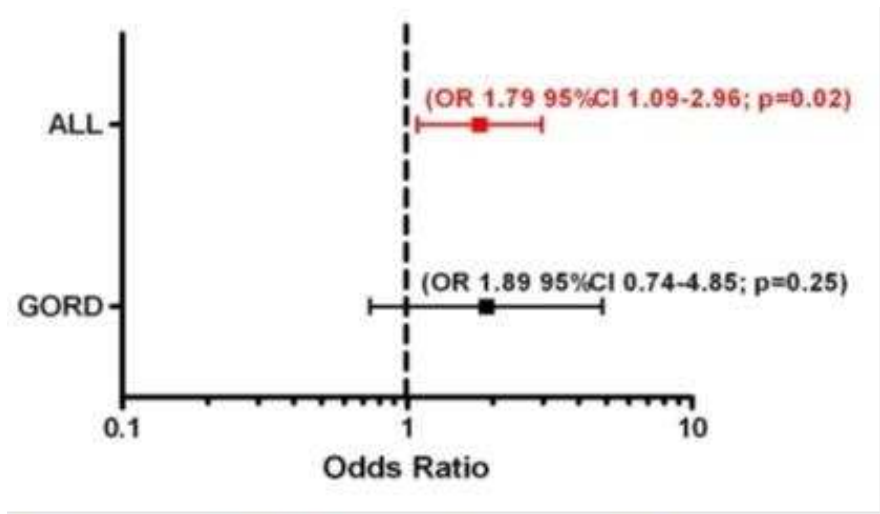

Figure 4: Correlation of Pepsin Assay results with presence of respiratory symptoms in the whole group and in the sub group of children with reflux symptoms

Figure 4: Correlation of Pepsin assay results with presence of respiratory symptoms in the whole group and in the subgroup of children with reflux symptoms

Pepsin was quantified and compared with respiratory symptoms which showed that a significantly greater quantity of pepsin was associated with the presence of respiratory symptoms in children (Figure Five).

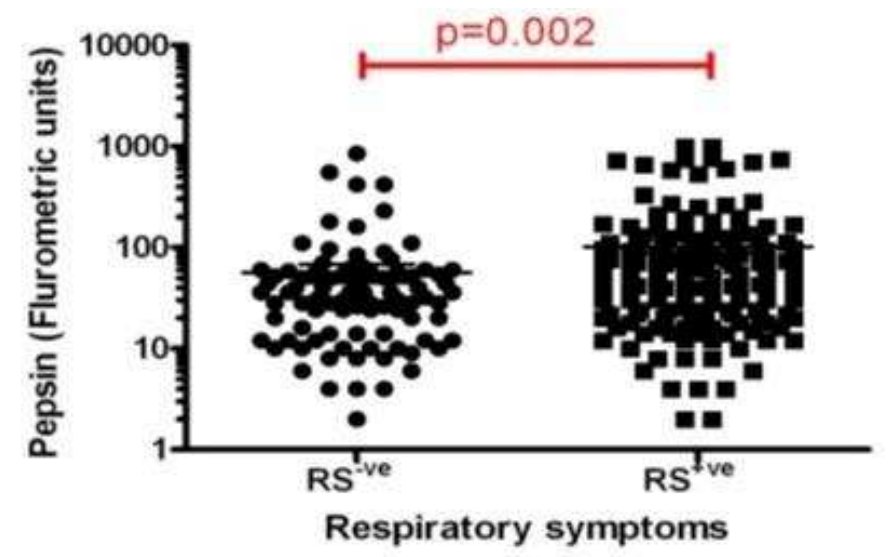

Figure 5: Quantification of Pepsin detected in children with and without respiratory symptoms

Figure 5: Quantification of pepsin detected in children with and without Respiratory symptoms

From our data, there was no statistically significant correlation for abnormal $\mathrm{pH}$ probe analysis, oesophagitis on biopsy or reflux episodes noted on barium meal and the presence of respiratory symptom. Due to the retrospective nature of this study, not all patients underwent every investigation; 64 had $\mathrm{pH}$ probe, 83 gastroscopy and 44 barium study. No correlation between respiratory symptoms and standard test results was identified. Positive pepsin assay was the only investigation found to be significantly associated with the presence of respiratory symptoms (OR 1.79, 95\% CI 1.09-2.96, p = 0.02); Figure Six. 


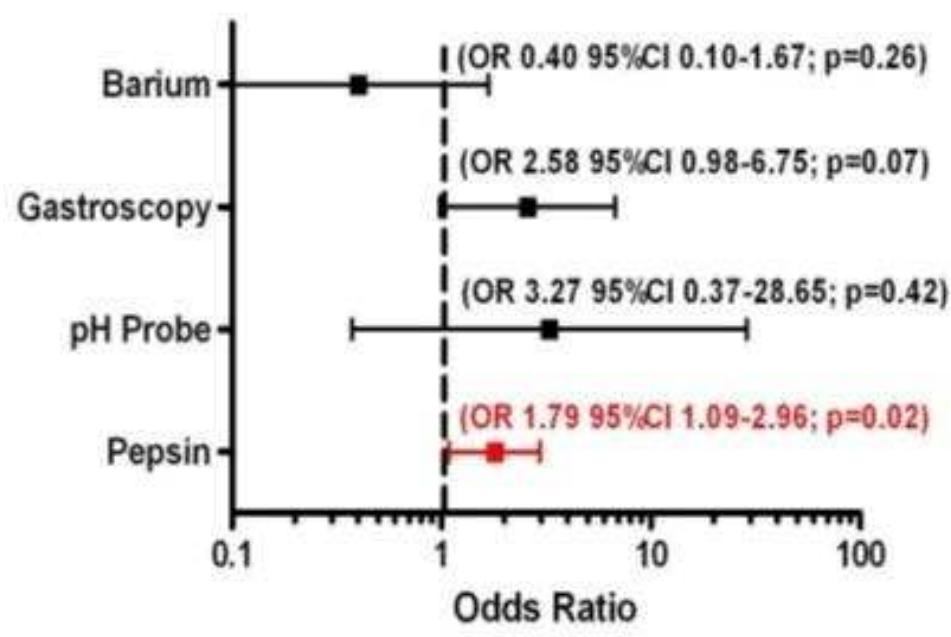

Figure 6: Comparison of Pepsin Assay results and results of standard investigations for GERD with presence of respiratory symptoms

Figure 6: Comparison of Pepsin assay results and results of standard investigations for GERD with presence of respiratory symptoms

Results from the clinician's questionnaire are summarised in Table Two.

Table 2: Questionnaire Results

\begin{tabular}{|l|c|c|}
\hline \multicolumn{1}{|c|}{ QUESTIONS } & YES & No \\
\hline QUESTION ONE: Did you find the results of the pepsin assay to be helpful? & $95 \%$ & $5 \%$ \\
\hline $\begin{array}{l}\text { QUESTION TwO: Did the results of the pepsin assay lead to an alteration in } \\
\text { the treatment of your patient? }\end{array}$ & $35 \%$ & $66 \%$ \\
\hline $\begin{array}{l}\text { QUESTION THRE: Did the results of the assay result in alteration of the } \\
\text { anti reflux medications that the patient was on? }\end{array}$ & $22 \%$ & $78 \%$ \\
\hline $\begin{array}{l}\text { QUESTION FouR: Did the results of the assay result in a fundoplication } \\
\text { being performed on the patient? }\end{array}$ & $10 \%$ & $90 \%$ \\
\hline $\begin{array}{l}\text { QUESTION FIVE: Did you obtain any new diagnostic information from this } \\
\text { assay which was not previously available? }\end{array}$ & $80 \%$ & $20 \%$ \\
\hline
\end{tabular}

148 of the 318 questionnaires sent out to clinicians were received back - giving a response rate of $47 \%$. There was an $82 \%$ response rate from gastroenterologists and a $23 \%$ response rate from other clinicians. The response to the first question showed that $95 \%$ of responding clinicians found the pepsin assay to be helpful in the treatment of their patients. $35 \%$ of respondents stated that the pepsin assay led to an alteration in patient management (Q2). $22.3 \%$ of clinicians responded that the pepsin assay result caused a change in the anti reflux medications the patient was prescribed (Q3). 10\% of the responding clinicians found that the pepsin assay result 
led to fundoplication being performed for their patient (Q4) and $80 \%$ of clinicians felt that the assay provided new diagnostic information for their patient, which was not previously available from the results of the standard investigations performed (Q5).

\section{Discussion}

Aspiration related to gastroesophageal reflux disease (GERD) is known to cause both upper and lower respiratory tract symptoms in children. Reflux aspiration can induce bronchospasm and may result in recurrent chest infections ${ }^{8,9}, 10$. The diagnosis of pulmonary aspiration secondary to reflux remains a challenge to clinicians. The diagnosis of reflux aspiration, prior to the advent of the pepsin assay, was often made with a combination of clinical, laboratory and radiological tests, such as 24 hour $\mathrm{pH}$ probe monitoring, barium swallow/meal and gastroscopy with biopsies. These tests can be indicative of GERD, but, unlike the pepsin assay, are not direct markers of reflux aspiration. The ideal test for reflux aspiration involves the detection of a marker within the lungs that should not be present within the tracheobronchial tree under normal conditions. This marker should be easily recovered and reliably detected following reflux aspiration. Lipid laden macrophages assayed from bronchoalveolar lavage (BAL) samples were previously used as a direct marker of reflux aspiration. This assay was found to be neither specific nor sensitive $^{4,3}$, and is not able to differentiate between direct aspiration from reflux aspiration ${ }^{13}$.

Pepsin in the lungs has been proposed as a biomarker for reflux related lung disease. Pepsin assay has the potential to significantly improve the sensitivity and specificity of the current diagnostic approach to reflux aspiration in the clinical setting. Our group was one of the first to look at pepsin as a marker of reflux aspiration in children with GERD ${ }^{4}$. Subsequently a link between the detection of pepsin in BAL or tracheal aspirates and reflux aspiration has been demonstrated in several human and animal studies $11,12,31$.
This study showed no statistically significant difference in the prevalence of pepsin positivity among children with either positive or negative 'standard investigations' (pH probe, barium contrast radiography and gastroscopy results). A recent study by Rosen et al correlating pepsin assay results to the results of $\mathrm{pH}$ impedance monitoring and gastroscopy found results that were similar to our study with respect to gastroscopy; however the number of non-acid reflux events on MII testing did correlate with pepsin positivity 30. This might be because MII is superior to $\mathrm{pH}$ monitoring in detecting GERD as it gives information on both acid and non acid reflux episodes ${ }^{32}$. The lack of correlation of pepsin results with gastroscopy is because endoscopy allows macroscopic assessment of the gastrointestinal tract while biopsy can demonstrate mucosal inflammation when present but it is not able to exclude reflux aspiration 14,15. Kaufman et al 16 noted that the amount of acid exposure required to cause respiratory mucosal inflammation is far less than that required to cause gastrointestinal mucosal inflammation. Further, it has been shown that the presence of pepsin in non-acidic refluxate can still cause mucosal damage when aspirated 17, 18.As such, even relatively infrequent reflux aspiration events may result in pulmonary irritation and inflammation without causing endoscopically visible evidence of GERD or abnormal pH study results. The pepsin assay is capable of detecting pulmonary presence of pepsin even in cases of infrequent reflux aspiration.

This study looks at the role of pepsin assay in the clinical management of children with suspected extra esophageal reflux disease (EORD). In this study although overall 50\% of the respiratory secretions collected tested positive for pepsin, of the specimens collected during a bronchoscopy, 76\% were pepsin positive and children having an LB for the investigation of chronic respiratory symptoms was 5.4 times more likely to test positive for pepsin compared to those who were having a gastroscopy for GERD symptoms. This result is different to the Rosen study where only $44 \%$ of the 
specimens collected during bronchoscopy were pepsin positive. We postulate that this difference could be because, in the Rosen study all patients who presented to bronchoscopy for evaluation of reflux disease had samples collected for pepsin assay and their sampling was not limited to those with both reflux and respiratory symptoms. Our results were comparable to the Rosen study when they limited their sample size to patients with both respiratory and abdominal symptoms, when the sensitivity and specificity of the pepsin assay increased to $70 \%$ and $81 \%$ respectively.

In our study, children with respiratory symptoms were 1.8 times as likely to be positive for pepsin and have a significantly higher concentration of pepsin when compared to children without respiratory symptoms. This significance held true even when comparing the pepsin positivity between the groups with respiratory symptoms to those with reflux symptoms alone. In this study, patients testing positive for pepsin were more likely to be affected by respiratory symptoms $(p=0.004)$. Of patients that were positive for pepsin, 58\% had accompanying respiratory symptoms. Positive pepsin assay was the only investigation which was significantly associated with the presence of respiratory symptoms. This finding was not replicated when compared with the 'standard investigations'. These results suggest that pepsin assay may be a superior marker for reflux aspiration resulting in chronic respiratory symptoms in children with GERD when compared to standard investigations such as $\mathrm{pH}$ probe, barium contrast radiography and gastroscopy.

The clinician questionnaire had a low (47\%) response rate, though it revealed interesting data. Clinicians involved in the study were able to use pepsin results in formulating appropriate management plans for their patient. Of all respondents, $95 \%$ found the pepsin assay to be useful and $80 \%$ found that the pepsin assay provided information on pulmonary aspiration that was not previously known. Both positive and negative pepsin results were found to be of use in clinical management, and alterations in medical management were made based on these results. As only $23 \%$ of patients were on anti reflux medications at the time of respiratory fluid sampling, a positive pepsin assay in a child who was not any anti reflux treatment led to the commencement of anti reflux medications in these patients, or optimisation of anti reflux treatment in those who were already on medications, either by increasing the doses of proton pump inhibitor (PPI) therapy, changing to twice a day PPI therapy, by the addition of prokinetics (erythromycin or domperidone), or by helping the clinician decide on the need for an anti reflux surgery. Positive results in symptomatic patients who had prior anti reflux surgery were thought to be indicative of unwrapping of prior fundoplication with consequent respiratory soiling by gastric secretions. Consequently, these patients were commenced on antireflux medications.

A negative pepsin result was also of help to clinicians to exclude reflux aspiration as a cause of chronic respiratory symptoms in a patient with GERD in whom a fundoplication was being considered due to the presence of chronic respiratory symptoms. A negative pepsin result was especially of value in neurologically impaired patients whose chronic respiratory symptoms are often secondary to direct aspiration, and not necessarily reflux aspiration. These neurologically impaired patients with a negative pepsin result, often subsequently had a video fluoroscopic swallow study, where the presence of direct aspiration led to a modification of their feeding either by the thickening of their feeds or gastrostomy placement.

Of responding clinicians, 15 of 148 had patients that proceeded to fundoplication following the results of the pepsin assay. In 5 patients from the study, a negative pepsin result prevented fundoplication from taking place. Of children who had previously undergone fundoplication, 10 from 18 tested negative for pepsin demonstrating effective fundoplication. 
Although the questionnaire responses indicated that management was altered based on the pepsin assay in only $35 \%$ of cases, this may be due to a negative pepsin assay result confirming that the patients' current anti reflux treatment regime was appropriate. The pepsin assay may also not have altered management in patients without evidence of respiratory compromise. Due to the retrospective nature of the study, not all alterations to clinical management were able to be noted on chart review, due to incomplete data being available. In addition, a $47 \%$ overall clinician response rate to the questionnaire meant that not all alterations to treatment based on the results of the pepsin assay were available to the authors.

The main drawback of our study was its retrospective nature with the associated limitations of incomplete data on some of the variables. In addition not all the clinicians responded to the questionnaire. Although an enzymatic assay was used for the assay of pepsin in this study, our group currently uses an ELISA based on a monoclonal antibody to human pepsin A, and has found good correlation between the enzymatic assay and the ELISA in the same samples. Pepsinogen $\mathrm{C}$ has been detected in lung tissue due, presumably, to lung injury, pepsin and pepsinogen $\mathrm{A}$ are not locally found in the lungs ${ }^{21,31}$. A recent study by Rosen et al 30 also found no evidence of pepsinogen A in bronchoscopy samples that were pepsin positive.

The strengths of this study lie in the large sample size, clinical correlation and information available directly from treating clinicians, which show that pepsin results

\section{References}

1. Kauer WK, Stein HJ, Möbius C, Siewert JR. (2004). Assessment of respiratory symptoms with dual $\mathrm{pH}$ monitoring in patients with gastro-esophageal reflux disease. Br J Surg. 91(7):867-71.

2. Paediatric Otolaryngology: Laryngopharyngeal Reflux (LPR). (2004). Retrieved June 22011 from http://www.pediatric- were found useful and in some cases, altered patient management. This is the first study to investigate the clinical role of the assay in the management of children with GERD in whom reflux aspiration is suspected. The joint ESPGHAN-NASPGHAN clinical practice guidelines on the management of GERD ${ }^{20}$ showed that pepsin assays of bronchoalveolar lavage fluid were an indicator of gastroesophageal related aspiration with Level B evidence. We hope that our study will provide further validation of the pepsin assay in the routine assessment of children with GERD and respiratory symptoms in who reflux aspiration is suspected. Further prospective outcome studies are necessary to determine if the modification of medical management and/or anti reflux surgery based on results of pepsin assay result in improved clinical outcomes in patients with reflux related lung disease. There is also a need to do prospective studies looking at the correlation between pepsin positivity and, not just the number of acid and non-acid reflux episodes and proximal migration of reflux episodes, but also symptom association indices using impedance testing in patients with chronic respiratory symptoms thought to be secondary to EORD. Studies comparing the results of pepsin assays performed via tracheal aspirates and BAL fluid with those done in saliva, exhaled breath condensate, sputum or nasopharyngeal secretions will help determine whether respiratory secretions obtained without intubation and tracheal suctioning would be adequate surrogate samples for pepsin assay in the future.

ent.com/learning/problems/laryngop haryngeal.htm.

3. Schwarz S.M. (2010) Gastroesophageal Reflux. Pediatric Gastroenterology, eMedicine. Retrieved online from http://emedicine.medscape.com/articl e/930029-overview

4. Krishnan U, Mitchell JD, Messina I, Day AS \& Bohane TD. (2002). Assay of tracheal pepsin as a marker of reflux aspiration. Journal of Pediatric 
Gastroenterology and Nutrition. $35 \mathrm{pp}$ 303-8.

5. Samuels et al. (2010). Pepsin as a marker of extraesophageal reflux. The Annals of Otology, rhinology and Laryngology. 119; 3 p 203.

6. Twining S.S. (1984). Fluoroscein isothiocyanate- labelled casein assay for proteolytic enzymes. Analytical Biochemistry. 143(1), pp30-4.

7. Vandenplas Y, Sacre-Smith L, Continuous 24-hour esophageal pH monitoring in 285 asymptomatic infants 0-15 months old. J Pediatr Gastroenterol Nutr. 1987; 6: 220-24.

8. Chen PH, Chang MH \& Hsu SC. (1991). Gastroesophageal reflux in children with chronic recurrent bronchopulmonary infection. Journal of Pediatric Gastroenterology and Nutrition. 13, pp16-22.

9. Jack CI, Calverley PM, Donnelly RJ, Tran J, Russell G, Hind CR \& Evans CC. (1995). Simultaneous tracheal and esophageal pH measurements in asthmatic patients with gastroesophageal reflux. Thorax, 50, pp10991108.

10. Varkey B, Pathial K, Shaker R. (1992). Pharyngesophageal reflux index in asthmatics. Chest. 102, pp152A.

11. Farrell S, McMaster C, Gibson, D, Shields, MD, McCallion WA. (2006). Pepsin in bronchoalveolar lavage fluid: a specific and sensitive method of diagnosing gastro-esophageal refluxrelated pulmonary aspiration. $J$ Pediatric Surger. .41(2):289-93.

12. Ufberg JW, Bushra JS, Patel D et al. (2004). A new pepsin assay to detect pulmonary aspiration of gastric contents amoung newly intubated patients. American $J$ Emerg Med. 22:612-14.

13. Krishnan U. (2002a). Fat laden macrophages in tracheal aspirates as a marker of reflux aspiration: A negative report. Journal of Pediatric Gastroenterology and Nutrition. 35, pp309-13.

14. Gillett P \& Hassall E. (2000). Pediatric gastrointestinal mucosal biopsy. Special considerations in children. Gastrointestinal Endoscopy Clinics of North America.10; pp 669-712.

15. Dahms BB. (2004).Reflux esophagitis: sequelae and differential diagnosis in infants and children including eosinophilic esophagitis. Pediatric and Developmental Pathology, 7, pp 5-16.

16. Kaufman JA, Belafsky PC, Bach KK, Daniel E \& Postna GN.(2002).Prevalence of esophagitis in patients with $\mathrm{pH}$ documents laryngopharyngeal reflux. Laryngoscope.112, pp 1606-9.

17. Johnston N, Wells C, Samuels T, Blumin. (2010). Rationale for targeting Pepsin in the treatment of Reflux Disease. Annals of Otology, Rhinology and Laryngology. 119(8): 547-558.

18. Starosta V, Kitz R, Hartl D et al. (2007). Bronchoalveolarpepsin, bile acids, oxidation and inflammation in children with gastroesophageal reflux disease.Chest. 132:1557-64.

19. Ervine E, McMaster C, McCallion W, Shields MD (2009). Pepsin measured in induced sputum, - a test for pulmonary aspiration in children? J Pediatric Surg. 44(10):pp 1938-41.

20. Pediatric Gastroesophageal Reflux Clinical Practice Guidelines: Joint recommendations of the North American Society for Pediatric Gastroenterology, Hepatology and Nutrition (NASPGHAN) and the European Society for Pediatric Gastroenterology, Hepatology and Nurtition (ESPGHAN). Journal of Pediatric Gastroenterology and Nutrition. 49; 498-547.

21. Foster $C$ et al. Pepsinogen C: a type 2 cell- specific protease. Am J Physiol 
lung cell Mol Physiol. 2004; 286:L3827.

22. Blondeau K et al. Gastro-oesophageal reflux and gastric aspiration in lung transplant patients with or without chronic rejection. Eur Respir J. 2008; 31:707-13.

23. Farhath $\mathrm{S}$ et al. Pepsin, a reliable marker of gastric aspiration is increased in tracheal aspirates from preterm infants who deevlop bronchopulmonary dysplasia. Pediatrics 2008. 121:e253-9.

24. Gopalareddy V et al. Assessment of the prevalence of microaspiration by gastric pepsin in the airway of ventialted children. Acat Pediatr. 2008;97:55-60.

25. Johnston $\mathrm{N}$ et al. Pepsin and carbonic anhydrase isoenzyme III as diagnostic markers for laryngopharyngeal reflux disease. Layngoscope. 2004; 114:212934.

26. FarrellS. McMaster $C$ et al. Pepsin in bronchoalveolar lavage fluid: a specific and sensitive method of diagnosing gastroesophageal reflux-realted pulmonary aspiration. J Pediatr Srug. 2006;41:289-293.
27. Vandenplas $Y$, Badriul $H$, Verghote $M$ et al. Esophageal $\mathrm{pH}$ monitoring and reflux esophagitis in irritable infants. Eur J Pediatr. 2004; 163: 300-4.

28. Lundell LR, Dent J, Bennett JR, et al. Endoscopic assessment of oesophagitis: clinical and functional correlates and further validation of the Los Angeles classification. Gut. 1999; 45: $172-80$.

29. Liacouras CA, Ruchelli E. Eosinophilic esophagitis. Curr Opin Pediatr. 2004; 16(5): 560-66.

30. Rosen R, Johnston N, Hart K et al. The presence of pepsin in the lung and its relationship to pathologic gastroesophageal reflux. Neurogastroenterol Motil. 2012. Feb; 24(2):129-e85.

31. Gerson KD, Foster CD, Zhang $\mathrm{P}$ etal . Pepsinogen $\mathrm{C}$ proteolytic processing of surfactant protein B. J Biol Chem. 2008; 283:10330-8.

32. Rosen R, Nurko S. The importance of mulitchannel intraluminal impedance in the evaluation of children with persistent respiratory symptoms. Am j Gastroenterol. 2004;99:2452-8. 